\title{
Théâtre et récit : l'exemple des pièces monologuées contemporaines
}

Theater and Narrative: The Example of Contemporary Monologic Plays

\section{André Petitjean}

\section{(e) OpenEdition}

Journals

\section{Édition électronique}

URL : http://journals.openedition.org/pratiques/5859

DOI : $10.4000 /$ pratiques.5859

ISSN : 2425-2042

\section{Éditeur}

Centre de recherche sur les médiations (CREM)

\section{Référence électronique}

André Petitjean, «Théâtre et récit : l'exemple des pièces monologuées contemporaines », Pratiques [En ligne], 181-182 | 2019, mis en ligne le 30 juin 2019, consulté le 22 juillet 2019. URL : http:// journals.openedition.org/pratiques/5859; DOI : 10.4000/pratiques.5859

Ce document a été généré automatiquement le 22 juillet 2019

(c) Tous droits réservés 


\title{
Théâtre et récit : l'exemple des pièces monologuées contemporaines
}

Theater and Narrative: The Example of Contemporary Monologic Plays

\author{
André Petitjean
}

\section{Introduction}

1 Depuis quelques décennies, on assiste à une véritable vogue de la parole solitaire au théâtre sous la forme de pièces insérées dans des recueils (Chambres de P. Minyana [1993]; Monologues de F. Billetdoux [1996]) ou d'œuvres autonomes : La Nuit juste avant les forêts de B.-M. Koltès (1988), Le Petit Bois d'E. Durif (1990), The Great Disaster de P. Kermann (1999), Face à la mère de J.-R. Lemoine (2006), Le Mardi à Monoprix d'E. Darley (2009), pour ne prendre que quelques exemples. Cet intérêt pour le monologue théâtral est partagé par de nombreux metteurs en scène ou comédiens qui, comme le rappelle H.-T. Lehmann (2002), parlant de «monologies", privilégient la mise en forme monologuée de drames classiques ou de textes narratifs (Heiner Goebbels, Prometheus ou le débarquement désastreux [1993], Robert Wilson, Hamlet -A monologue [1994]) ainsi que les solis performés par des acteurs seuls en scène (M. Caubère et les 11 épisodes du Roman d'un acteur, 1993; R. Lepage, et ses cinq spectacles solo dont Elseneur, 1997). À l'évidence, ces formes dramatiques sont loin de respecter l'ordonnancement du «bel animal» selon les principes aristotéliciens (unicité de l'intrigue, progression ordonnée et finalisée des actions, développement temporel chronologique, causalité explicite des enchainements). C'est que raconter sa vie est plutôt antidramatique pour au moins deux raisons. D'une part, le drame, comme l'a théorisé Aristote, est avant tout action et non pas narration par l'intermédiaire de personnages qui agissent en parlant. D'autre part, la contrainte du passage à la scène nécessite un effort de concentration dramatique qui, à la différence du roman, rend difficile l'extension de la diégèse à la totalité d'une vie. L'une des solutions pour dépasser ces contradictions est de recourir à un personnage qui parle et raconte, 
assumant, de ce fait, son double rôle de "figure narrante » et de "personnage narré " sous la forme du "personnage souvenant ». En ce sens, les "pièces monologuées » sont assez emblématiques de textes dans lesquels le récit prend volontiers le pas sur l'action.

Il me semble que pour mieux comprendre le statut de ces pièces, il n'est pas inutile de rappeler les caractéristiques des monologues classiques, en particulier dans les tragédies. J. Scherer (1950) ou C. Triau (2009), sans mésestimer le fait que le monologue a changé de statut au cours du $\mathrm{XVII}^{\mathrm{e}}$ siècle, relèvent les fonctions suivantes: informative (apport d'informations diégétiques nécessaires à la compréhension de l'intrigue), expressive ou vocative (dévoilement lyrique, voire pathétique des sentiments ; interpellation d'un objet ou d'un sentiment personnifié), délibérative (exposition d'un choix alternatif dans le monologue dit de « dilemme »), emblématique (identification du personnage à son imago mythologique, sur le mode de la confirmation ou de la transgression), dramaturgique (commentaire de l'action, transition entre les scènes). À quoi s'ajoute le fait que le monologue permet de mettre en valeur la virtuosité déclamatoire de l'acteur. Je retiens, enfin, que le monologue est à rapprocher, dans la dialogie interne, de la tirade, des stances et de l'aparté. Si le monologue de la dramaturgie classique se soumet aux exigences de la vraisemblance et de l'interlocution avec les expérimentations symbolistes puis surréalistes jusqu'aux pièces contemporaines, le monologue va progressivement transformer les modes de composition du « drame absolu » de P. Szondi (1983).

Pour le montrer, j'ai retenu trois phénomènes qui caractérisent ces pièces : le narratif, le dialogisme et le poétique. C'est ainsi que dans un premier temps, je montrerai en quoi ces textes sont de véritables récits, car la fable, proche en cela des fictions romanesques, n'est plus soumise au développement temporel strictement chronologique. Grâce à une alternance entre narration et monstration, le personnage peut voyager en permanence dans des chronotopes différents, passés, présents voire à venir.

Dans un deuxième temps, on verra que la solitude du personnage n'est pas forcément antidramatique puisque ces monologues sont très polyphoniques. Leur intérêt est de faire advenir de nombreux personnages et c'est là un indice du processus de romanisation que connait le théâtre depuis plusieurs années. En effet, le personnage narrant fait entendre une multitude de voix, que ce soit les siennes à différentes époques de sa vie ou celles d'autres personnages qui peuplent son existence. Ce dialogisme est à la fois interlocutif (le personnage s'adresse à un autre en l'interpellant, voire au spectateur), interdiscursif (voir les paroles rapportées des personnages au cours de la narration de scènes passées) ou autodialogique (voir les boucles réflexives au cours desquelles le personnage revient sur ses propos tenus).

5 Pour finir, je montrerai que ces trois pièces, à des degrés divers, possèdent un rythme interne particulier qui tient à l'usage de la répétition dont les effets sont à la fois élégiaques et mélodiques.

6 Afin de ne pas me disperser, j'ai limité mon corpus à trois pièces (La Nuit juste avant les forêts, Face à la mère, The Great Disaster).

\section{La narration}

7 Au niveau de leur composition, ces genres de pièces que J.-P. Sarrazac (1989) qualifie de «monologue-confession », sont de longues coulées verbales en forme de récit (63 pages pour B.-M. Koltès, 54 pages pour J.-R. Lemoine et 45 pages pour P. Kermann). Dans tous les 
cas, le personnage-narrateur s'adresse à un interlocuteur au statut différent. Chez B.M. Koltès il est présupposé présent mais silencieux, comme en témoigne l'omniprésence des pronoms d'adresse «tu » à l'ouverture de l'incipit et continûment réactivée par la suite (« tu tournais, là-bas, le coin de la rue, lorsque je t'ai vu » [p. 7]; « je vois bien que tu ne te méfies pas, tout petit nerveux que tu es, tu ne te méfies pas » [p.18]). Avec J.R. Lemoine, le monologue commence par un prologue au cours duquel il est stipulé que le texte qui va suivre est adressé à la mère disparue, assassinée par les sbires de Duvalier à Haïti. Effectivement, à différents moments, l'adresse est explicite : «Vous me manquez, maman, vous me manquez. Je voudrais que vous soyez-là »(p.17), «Mère, je vous pardonne. Et je vous demande pardon » (p.56). Mais dans le prologue, il est souligné méta-théâtralité oblige -, qu'il sera écouté par les récepteurs additionnels que sont les spectateurs («Il aura fallu trois années de parenthèse, trois années de coma profond, pour pouvoir vous donner rendez-vous dans ce lieu ombragé, devant l'assemblée silencieuse ", p. 12). Chez P. Kermann, en l'absence d'adressé direct, on suppose que le monologue est dirigé vers le récepteur additionnel sans que l'on puisse en être certain. Dans tous les cas, les personnages sont inscrits dans un présent mimétique à partir duquel ils décrochent dans des chronotopes différents. Pour B.-M. Koltès, le cadre spatiotemporel immédiat dans lequel est situé le personnage-locuteur est assez indéterminé au point que les critiques divergent pour sa localisation. Alors que J.-P. Sarrazac (1996) et A. Ubersfeld (1999) penchent pour le « coin d'une rue ", F. Benhamou (2001) le situe à l'intérieur d'un «café ». Le présent est le temps principal qui fait système avec les déictiques temporels tels que " maintenant» (13 occurrences) et plus occasionnellement « à présent » et « aujourd'hui ». Avec « maintenant » l'empan temporel de référence est plus ou moins étendu pouvant être paraphrasé en "au moment où je te parle » (« maintenant qu'on est là », p. 7) et « de nos jours » (« maintenant je ne travaille plus », p. 31). Remarquons le nombre non négligeable de «tout à l'heure » (6 occurrences) qui renvoient, selon les emplois, à un passé ou un futur proche («lorsque je me lavais tout à l'heure ", p. 11 ; " on ira tout à l'heure voir où c'était ", p. 42). Le locuteur se livre à une intense activité de commentaire qui prend appui, outre l'omni présent d'énonciation («alors moi, j'ai cette idée, camarade pour ceux comme toi et moi qui n'avons pas d'argent ni de travail», p. 14) sur le futur et le conditionnel. Le premier manifeste l'activité de conjecture du locuteur sur son devenir, que ce soit au niveau de sa condition matérielle (« dès qu'on sera installé quelque part, je m'enlèverai tout c'est pour cela que je cherche une chambre ", p. 8) ou de ses projets politiques ("jusqu'à ce que mon idée de syndicat international ait fini par gagner [...] ce sera aux rats de jouir, camarade, ce sera à notre tour ", p. 26). Le second est lié aux énoncés irréels ou potentiels qui sont autant de traces d'une réflexion raisonnante. Elle prend la forme de suppositions (« on me donnerait une sorte de petite chaumière [...] j'y entrerais, moi, avec rien du tout et en un rien de temps », p. 10), de contrefactualité (« il faudrait être ailleurs, personne autour de soi, plus question d'argent et cette saloperie de pluie », p. 47); d'hypothèse (" - si elle avait fermé sa gueule, je n'aurais jamais su ce qu'une gueule comme cela », p. 22). Quant au site spatial, le locuteur y réfère à l'aide des déictiques, principalement "ici » (18 occurrences) et secondairement « là » (3 occurrences) : " (s'il n'y avait pas la question de l'argent), de se barrer d'ici (si on savait où aller) d'être dans une chambre, vieux, où je puisse parler, ici je n'arrive pas à dire ce que je dois dire ", p. 47) ; " et maintenant qu'on est là, que je ne veux pas me regarder», p.7). Il est vrai qu'ils n'ont pas la même référence selon qu'ils sont employés en énonciation primaire ou rapportée (" tu ne lui dis pas ton nom, tu lui dis : où on va ? elle te dit: où tu voudrais aller ? on reste ici, non?", 
p. 34). Le déictique « ici » est en fait à géométrie variable si bien que selon le contexte de son emploi, on infère qu'«ici » signifie le pays, le quartier, la rue ou le café avec ses toilettes au sous-sol : « je ne suis pas tout à fait d'ici - c'était bien visible en tout cas [...] à croire qu'ils sont tous aussi cons les Français, incapables d'imaginer », p. $10 ;[. .$.$] pourtant$ c'en est plein comme partout ici, jusque dans les hôtels cent mille glaces vous regardent dont il faut se garder », p. 8 ; «il est difficile de ne pas se regarder, tant par ici il y a de miroirs, dans les cafés, les hôtels, qu'il faut mettre derrière soi, comme maintenant qu'on est là », p. 8 ; «je t'ai vu tourner le coin de la rue [...] il ne fait pas bon de tourner ce soir par ici, pour toi comme pour moi », p. 12 ; «malgré tous les cons qu'il y a dans la rue, dans les cafés, dans les sous-sols de café, ici, partout», p. 12 ; « et je suis remonté - juste le temps de pisser - avec mes fringues mouillées ", p. 7. Il est vrai, enfin, que le locuteur contribue à nous faire une idée des lieux en présence à l'aide de brefs segments descriptifs introduits par le démonstratif: «toi, moi, qui tournons dans cette drôle de ville» (p. 14); «il ne fait pas bon de tourner ce soir par ici (p. 12); « un soir comme ce soir » (p. 41) ; « malgré cette saloperie de pluie et de lumière triste » (p. 28).

Convenons d'appeler espace-temps diégétique les réalités fictionnelles non visualisées dans le cadre mimétique et qui élargissent ce dernier à des horizons antérieurs et passés. En effet, dans ce "théâtre narration", grâce à une alternance entre narration, monstration (dialogues rapportés) et délibération (commentaires) on voyage en permanence dans le temps et l'espace. Dès l'instant où le locuteur, à l'incipit et à l'excipit de la pièce, tient des propos similaires quant à sa situation, on comprend que l'essentiel $\mathrm{du}$ soliloque se déroule le temps d'une rencontre relativement brève ( $«-$ avec mes fringues mouillées je resterai comme cela, jusqu'à être dans une chambre» [p. 7]; «j'ai envie d'une chambre et je suis tout mouillé »[p.63]). Dans l'intervalle, le locuteur interrompt sa parlerie par une multitude de retours analeptiques dans des chronotopes diégétiques différents plus ou moins éloignés du présent de l'énonciation. Cela tient au fait que le locuteur est aussi un narrateur qui, par le biais d'une biographie embryonnaire, fait retour sur son passé. Il opère à l'aide de microrécits dont les contenus, sur le mode des "souvenirs écrans", sont de façon indécidable réels ou fantasmagoriques. Parfois itératif («chaque nuit» [p. 28], «lorsque je travaillais» [p. 31]), ces récits sont plutôt singulatifs («un soir, par hasard» [p. 34]). Au niveau temporel, les éléments de cadrage sont indéterminés quand ils réfèrent à un passé éloigné (« lorsque je l'ai vu » [p. 30], « lorsque je travaillais » [p. 31]) et bornent assez précisément le déroulement temporel qui précède la rencontre («dans la journée» [p. 36], « dès le début de la soirée » [p. 56]). Au niveau spatial, à l'opposé de l'« ici » mimétique, « là-bas » est le marqueur caractéristique de ces différents lieux (" eux ils finissent ici et moi je finirai là-bas, - là-bas où tout ce qui bouge est caché »[p.51]). Ces microrécits, à l'exception de l'allusion au Nicaragua (p.48-52), concernent la vie passée du locuteur qu'ils évoquent non pas selon un ordre chronologique mais au gré d'un flux de conscience désordonné. C'est ainsi que la «ville» dont on peut inférer qu'elle représente Paris est convoquée quand il évoque tour à tour, la rencontre avec la fille blonde dans un café (p. 20-26), le travail à l'usine (p. 19), l'épisode de Mama au bord du fleuve (p. 34-36), la pute du cimetière (p. 37-38), le quartier chaud et la pute du quatrième étage (p. 41-42 et 45-46). À quoi s'ajoutent différents moments de la soirée : la bagarre avec les loubards (p.56), l'errance dans le métro (p.60), la pluie et la recherche d'un hôtel (p.7), l'attroupement dans les toilettes au sous-sol d'un café (p. 7 et 10 et 11). Remarquons que le locuteur revient aussi à plusieurs reprises sur le moment de sa rencontre avec 
l'allocutaire (p. 7, 8, 12, 15, 30,32) et qu'il fait allusion tour à tour à la planification des "salauds techniques» (p.19) et à son projet de syndicat international (p. 26, 27). L'ensemble est dominé par une vision dysphorique (la nuit, la pluie, la solitude). Notons que B.-M. Koltès s'emploie à rendre étrange les lieux qui nous sont familiers tels qu'ils sont fréquentés par des personnages marginaux et énigmatiques (la «fille sur un pont [p. 34] ou « cette cinglée de pute» [p. 38]). Ces épisodes diégétiques sont au niveau des tiroirs verbaux pris en charge par le passé composé historique combiné à l'imparfait ( «j'avais assez d'argent pour toute la soirée à boire autant de bières que tu l'aurais voulu et pour être o. k., mais ils me l'on piqué » [p. 57]). Dans certains récits, les faits sont racontés en forme d'hypotypose au présent de narration : " comme cette femme-là qui $y$ est bien passée d'avoir avalé de la terre, elle va dans le cimetière, elle creuse à côté des tombes, elle prend la terre dans ses mains, la terre la plus profonde, et l'avale - » (p. 38).

Chez J.-R. Lemoine, le monologue est divisé en trois « mouvements » pendant lesquels le récitant, un homme, sans patronyme, revient sur son passé. En témoignent les occurrences multiples des verbes «se souvenir» (« Si je me souviens bien, ce fut dans un théâtre où avait lieu un atelier de comédiens » [p.13], «Si je me souviens bien, jusqu'à l'âge de vingt ans j'ai été votre fils » [p. 31]). À quoi s'ajoutent les allusions à l'activité de mémoration ("Et toujours la même scène qui revient à la mémoire » [p. 43], «Gratter la mémoire jusqu'à l'os » [p. 27]). Il le fait à l'aide d'un effort de mémoire ou avec l'appui de photographies et de la correspondance familiale ( Cette maison qui était la maison de ma mère, avait été, avant, celle de mes grands-parents. Je m'asseyais par terre au milieu des reliques. Les heures passaient dans le chapelet ininterrompu de la découverte d'une vie dont j'étais absent. Je lisais les lettres, les cartes postales, je regardais les photos, je parcourais les agendas de mon grand-père. » [p. 23]). Le but est de relater la relation à sa mère au travers d'épisodes de sa vie d'enfant et d'adolescent et de l'exil qu'ils ont connu, lui, sa sœur et sa mère, avec un père absent. Outre la description qu'il essaye d'accomplir de son état depuis que sa mère est morte trois années plutôt (p. 12), le monologue est un chant d'amour adressé à cette mère, sur fond de regret de ne pas avoir su et pu lui dire de son vivant combien il l'aimait malgré leurs différends. Inscrit dans un cadre spatiotemporel faiblement déterminé, le narrateur va raconter ce qu'il a vécu depuis ce jour funeste (p.13). On comprend que le moment où il décide de s'adresser à sa mère a lieu trois années après son décès ( Il aura fallu trois années de parenthèse, trois années de coma profond, pour pouvoir vous donner rendez-vous » [p. 12]) et on infère que la durée du chronotope mimétique est de trois mois. Il est fait allusion tour à tour à l'été puis à l'automne («Je vous écris dans la chaleur de l'été » [ibid.], « Ici l'été est lourd et chaud. Les journées sont immobiles dans l'attente de l'orage. Je mange des pêches blanches, je me couche tard, je parle peu, je lis quelques pages de roman, parfois une mouche entre par la fenêtre, tournoie désespérément, puis s'envole à nouveau vers l'azur» [p.34], «L'automne est arrivé soudain. Les brouillards parfois se dissipent. Les pluies sont souvent diluviennes. Le vent s'essouffle. Tout s'effiloche et se défait » [p. 55]). Le présent est le tiroir temporel dominant tel qu'il accompagne des commentaires expressifs ( $\mathrm{Je}$ n'ai plus d'horizon» [p. 29], «Je ne peux plus» [p.49], «Je ne sais plus» [p. 23]) ou réflexifs ( Parfois je me demande comment j’ai pu supporter cela. À chaque instant on croit qu'on ne pourra pas affronter l'instant supplémentaire et pourtant on survit " [p. 23]). Ils disent tous combien le travail de deuil est difficile à accomplir. Rares sont les déictiques spatiaux («Ici l'été est lourd et chaud» [p. 34]) ou temporels («Je me sens très seul maintenant » [p. 18], « Trois semaines ont passé et puis, un jour, dans ce théâtre, j'ai entendu les sanglots et la voix de ma sœur qui me disait que vous étiez morte » [p.28]) 
susceptibles de configurer le cadre mimétique. Le récit commence au moment où le récitant apprend la mort de sa mère (p. 13) et se poursuit par des scènes remémorées qui mettent en péril l'ordonnancement chronologique puisqu'elles sont entrelardées par des retours en arrière et une ellipse de plusieurs années. (« Dix années ont passé » [p. 25]). On assiste ainsi à différents épisodes qui comprennent l'enterrement de la mère à Sainte Rose de Lima (p. 15-17), l'enfance en Afrique (p. 19), l'arrivée en Belgique (p. 20), la suite du récit de l'enterrement (p. 22-24), le retour en arrière à Haïti dix ans auparavant (p. 25-26), l'enfance en Belgique (p. 31-34), les grandes vacances en Extrême-Orient pour rejoindre le père expatrié (p. 35), la continuation des années belges (p. 35-38), le retour à la journée de l'enterrement (p. 39), le séjour en France (p. 45), le retour à Fort-de-France (p. 46-50), puis en Belgique (p. 52). Les évènements sont racontés essentiellement au passé composé historique sur fond d'imparfait (« Nous avons ouvert les portes - persiennes et la lumière a envahi la maison. C'était la douce lumière du matin qui éclairait l'acajou des meubles et se posait en oblique sur les volutes chamarrées du carrelage" [p. 21]). Précédés parfois par des cadratifs temporels singulatifs («Un matin, après les funérailles », p. 39 ; «Un jour, je ne sais plus à quelle occasion» [p. 39]) ces moments alternent avec des scènes itératives (« Le dimanche, nous allions tous les trois à l'église où je vous regardais prier, puis vous nous accompagnez au cinéma. [...] Chaque trimestre, je vous apportais votre ration de résultats scolaires »[p. 33]). Une scène est inventée et non pas remémorée, celle où le personnage, en totale empathie avec sa mère, imagine comment elle a été assassinée. Elle prend alors la forme d'une hypotypose au présent de narration («Et toujours la même scène qui revient à ma mémoire, que je recrée, que je veux laisser passer, comme un nuage dans le ciel et qui revient encore, quelle heure estil ? combien sont-ils ? trois, peut-être quatre, ils entrent dans la maison. Elle se réveille. Elle entend les pas dans l'escalier. Elle se lève. Ils sont déjà devant elle [...] ils la frappent, elle se débat, elle est grande et forte malgré son âge... » [p. 43]). Ce faisant, J.-R. Lemoine brosse le tableau de régime dictatorial de Duvalier, président de la République d'Haïti de 1971 à 1986. Pour finir, un épilogue en forme d'adresse directe à la mère, permet au récitant, apparemment réconcilié et apaisé, d'accompagner en pensée sa mère outretombe.

10 Avec la pièce de P. Kermann, le titre en anglais (The Great Disaster) fait référence au naufrage du Titanic « le 14 avril 1912 à 23 heures 40 » comme le précise le récitant qui se dénomme Giovanni Pastore. Ce dernier, naufragé disparu avec le Titanic, raconte dans ce monologue d'outre-tombe son enfance dans les hautes montagnes du Frioul puis sa dure condition de travailleur immigré à travers la France, la Suisse et l'Allemagne, à la recherche d'un travail. Il finira par s'embarquer clandestinement sur le Titanic et faire la plonge à bord du bateau avant d'être englouti avec lui dans «les eaux glacées" de l'Atlantique Nord qui sont aussi celles « du calcul égoïste » comme l'aurait dit B. Brecht dans sa célèbre formule. En effet, P. Kermann transforme ce fait divers, qui a fait sensation à l'époque, en une fable libertaire au cours de laquelle se trouve confirmé le fait que «l'Histoire est avant tout celle de la lutte des classes» (B. Brecht encore). À la différence des deux pièces précédentes, le monologue de $\mathrm{P}$. Kermann, dans la mesure où le récitant est mort au moment où il parle, peut être considéré comme une "pièce épitaphe» (Sarrazac, 1989) sous la forme d'une sorte de hantise. Or, l'une des qualités essentielles des fantômes est de posséder un savoir en prise avec le passé, maitres qu'ils sont de la mémoire et des souvenirs.

elle avait quitté le village je n'avais pas dix ans

pour la ville 
on rentrait parfois ensemble car on habitait plus haut dans

la montagne

en été elle portait une jupe bleue

je m'en souviens

(p. 34)

moi Giovanni Pastore me souviens

pas de ma faute

- Giovanni a une mémoire phénoménale disait le curé à la Mamma si seulement il

voulait se donner un peu de peine il retient tout mais pas ce qu'il devrait.

(p. 14)

11 Cette faculté de mémoration du personnage lui permet de se souvenir des évènements dans leur moindre détail comme l'attestent ces longues listes au cours desquelles le récitant compte et décompte, non sans un certain humour noir parfois, les morts et les rescapés ou, à plusieurs reprises, les fournitures et les nourritures embarquées dans ce « paquebot de luxe » qu'est le Titanic, accomplissant ainsi sa fonction testimoniale :

ainsi

me souviens des 29 chaudières

des 800 caisses de noix

des 5 pianos à queue

des 8 douzaines de balles destinées à R.F. Downey et

Compagnie

des 15000 bouteilles de bière

des 7,7 tonnes de tomates

de la caisse de porcelaine pour Tiffany's de New-York

du trousseau d'Eleanor Widener qui allait se marier aux

States

de la petite boite métallique du major Peuchen (déjà

marié)

des 35000 œufs frais (quelle omelette)

de la machine à faire de la glace du pont $\mathrm{G}$

des 16 malles de Ryerson

des fauteuils d'osier du Café Parisien

des 180 caisses d'oranges et 80 de pamplemousse

etc. (p. 15)

12 Une autre qualité du fantôme traditionnel est son pouvoir d'abolir la distance entre le passé et le futur et de prédire l'avenir. C'est ainsi que Giovanni, mort, je le rappelle, le 14 avril 1912, a le don de voyance et de prévoyance. Il sait que Gatti, directeur d'un restaurant du Titanic par qui il s'est fait embaucher pour la traversée, a échappé au naufrage et s'est installé ensuite à New-York :

Car monsieur Gatti s'en est tiré lui

paraît même qu'il tient une pizzeria dans la $\mathrm{X}^{\mathrm{e}}$ Avenue

valait mieux mourir directeur (p. 13)

13 Giovanni annonce la fin de la Belle Époque et prévient que le capitalisme triomphant débouchera sur la boucherie de la guerre de 14 puis l'horreur des camps de concentration de la Seconde Guerre :

après la der des der on s'est dit

non ça ne peut pas continuer ainsi

des milliers et des milliers de soldats dans la boue

des jambes éclatées tripes à l'air

poumons abîmés

tranchées regorgeant de cadavres

vite vite on referme 
et en 45 quand ils sont revenus de là-bas

quelques-uns

squelettiques

le visage émacié

numéro tatoué sur le bras

avec des yeux vides d'avoir vu ce que personne n'aurait

imaginé de voir

l'horreur et pire que la mort

la fin d'une époque

enfin

c'est de cela que moi Giovanni Pastore rêve

car parfois je rêve

des choses anciennes

des choses qui ne se sont pas encore produites

je rêve

vestige de mon enfance dans les montagnes à garder

les brebis

je me lance tout droit dans des rêves sombres

que je ne peux raconter que la nuit. (p. 10)

Giovanni prophétise aussi, tel est son pouvoir de véri-diction, la période fasciste de l'Italie et son prolongement avec l'attentat de la gare de Bologne (1980) attribué à un groupe néo-fasciste.

même le Marcello

quand il est parti dans les montagnes prendre le maquis

il a abattu trois Allemands qui faisaient leurs bagages

ça compensait les Agneli que Roberto avait dénoncés pour

activités antifascistes

[...]

le fils Marcello

Giovanni comme moi

lui c'est carrément l'histoire nationale

Bologne la gare

des dizaines de morts des blessés partout

il y était

lui aussi a sa plaque. (p. 37)

15 Le cadre spatio-temporel immédiat dans lequel se profère le monologue, est plutôt déterritorialisé (« une voix (sortie) du fond de la mer »). Il faut bien admettre qu'il s'agit d'un «espace cérémoniel » qui ne saurait être figuré réalistement au moment de son incarnation par l'acteur. Le présent est le temps repère de cette énonciation personnelle ("Moi Giovanni Pastore adore les chiffres»; "Moi Giovanni Pastore me souviens»). Quant aux espace-temps diégétiques, on voit que le personnage voyage en permanence dans des chronotopes différents. P. Kermann, a planifié sa pièce en 10 séquences qui correspondent massivement à la nuit tragique du naufrage dont le récit se fait au présent historique avec des scènes parfois expansées en forme d'hypotypose :

Mais moi Giovanni Pastore étais là

tout vu tout entendu

les grondements sourds

les passagers qui tombent

les bousculades les coups de poings et de pistolets

et tout le navire qui chavire de l'avant

la cheminée qui se casse en deux dans un nuage

d'étincelles

pas le temps de chanter 
le vacarme et les craquements

des hommes qui se jettent dans les canots

la mer bouillonnante et tourbillonnante

à la verticale le Titanic se dresse

cul en l'air. (p. 44)

16 Au cours de son récit, le narrateur-fantôme prouve son omniscience extrahumaine en montrant qu'il est capable, comme le héros du Diable boiteux, de diégétiser différents espaces du navire pour surprendre les conversations des passagers voire de pénétrer dans leur conscience.

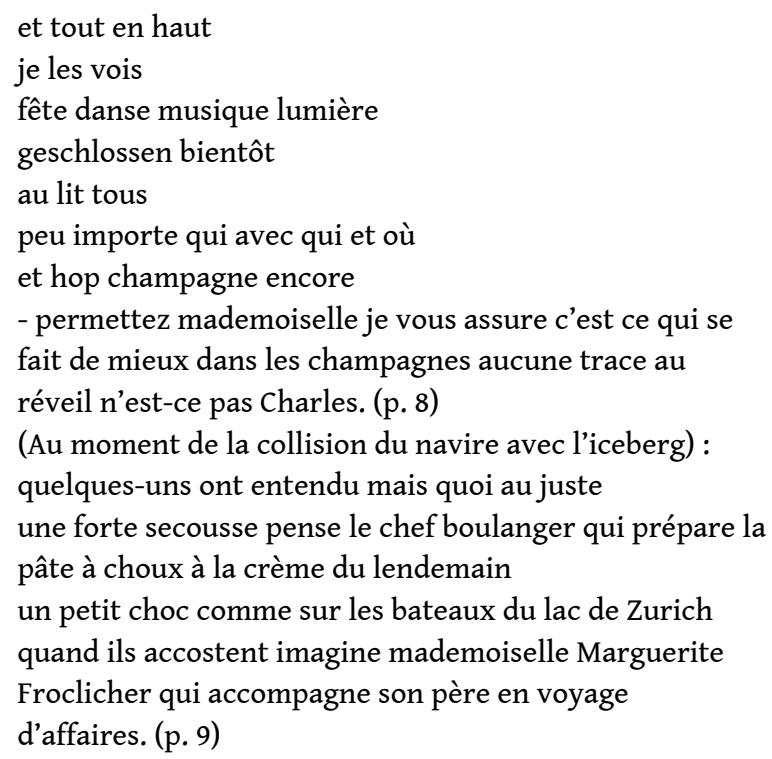

17 Le récit du naufrage est entrecoupé par de brèves excursions analeptiques selon qu'elles réfèrent au monde euphorique de l'enfance de Giovanni dans les montagnes du Frioul (séquences $1,3,4,5,8,9,10$ ), à son errance à travers l'Europe à la recherche d'un travail (séquence 5) et au jour de l'embarquement sur le Titanic lors de son escale à Cherbourg, (séquence 2). Elles sont énonciativement prises en charge par le passé composé historique + imparfait :

moi Giovanni Pastore

un jour je suis descendu de ma montagne

de mes verts pâturages aux sommets couverts de neige

moi Giovanni Pastore ai passé vingt ans de ma vie

à grandir dans les herbes folles

à pêcher dans le ruisseau les truites argentées

à suivre les papillons aux ailes bleues

à écouter le vent d'est qui amène les nuages de pluie

vingt ans

[...]

(p. 21)

Mamma je m'en vais Mamma je t'aime

alors elle m'a serré dans ses bras

[...]

et suis parti

sans me retourner

[...]

ai pris mon bâton ai marché

de bourg en bourg

de plus en plus nombreux sur les routes 


\section{[...]}

alors avons continué à marcher

des rumeurs des bruits guidaient nos pas

en France oui en France il y a du travail

[...]

la Suisse non plus d'ailleurs

n'ai fait que passer

rien que la moustache faisait trop sale

l'ai rasée

[...]

Gatti m'a dit à Cherbourg

d'homme à homme

- Giovanni je te prends ça ne sera pas facile mais je crois

que tu peux arriver à quelque chose surtout là-bas où

tout est à faire.

(p. 22, 23, 24, 25)

Les microrécits dont certains se répètent en boucle sont soumis à une intense activité commentative qui permet au récitant, par exemple, de traiter avec humour son destin tragique dès l'instant où il entre dans la légende en faisant la une de la presse et, du même coup, la fierté de la Mamma (p. 16).

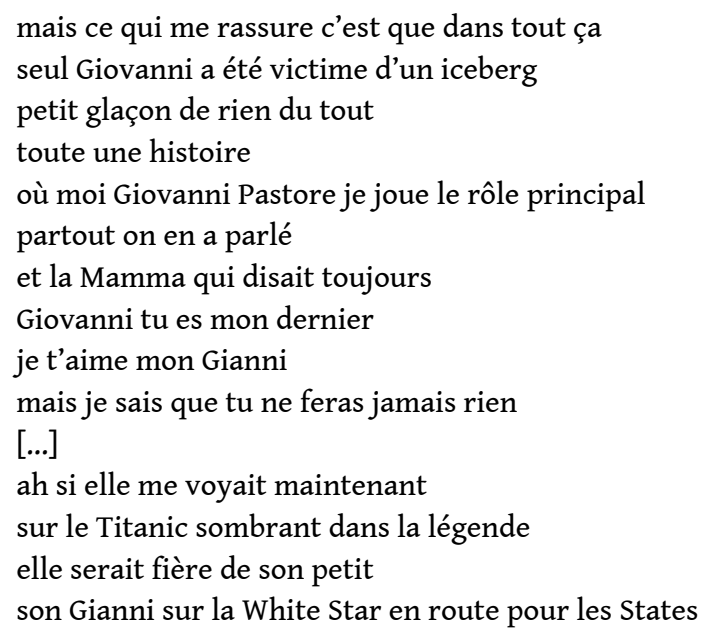

\section{Le dialogisme}

La solitude du personnage narrant, à la fois «souvenant» et "récitant » comme les appelle S. Beckett dans Solo et dans Cette fois, ne l'empêche pas de faire entendre une multitude de voix, que ce soit les siennes à différentes époques de sa vie ou celles des autres. Il s'ensuit que dans ces trois pièces la dimension dialogique (Bres, 2005) est importante, le personnage faisant entendre bien des « discours autres » (Authier-Revuz, 2004) qui se mêlent au flux continu de la voix du locuteur. Cette hétérogénéité est à la fois " montrée » à travers différentes formes de discours d'autrui et «constitutive " au sens où toute production verbale peut être traversée par des paroles ou des pensées autres. Le locuteur n'a pas nécessairement conscience de l'existence de ces voix (individuelles ou collectives) dont il se fait le porte-parole ventriloque. C'est en ce sens que J.-M. Piemme (1994) déclare, à juste titre : «On ne peut jamais réduire le monologue à quelqu'un qui parlerait seul. Certes l'acteur se trouve seul en scène mais il est dans une parole dialogique. C'est quelqu'un qui s'adresse à un autre ou prend en charge les paroles des autres. » 
Pour la pièce de B.-M. Koltès, en ce qui concerne le dialogisme "montré », il est d'abord interlocutif sous la forme de propos adressés directement à l'interlocuteur (« ne dis rien, ne bouge pas, je te regarde, je t'aime, camarade », p. 63). On s'arrêtera, par contre, sur le dialogisme interdiscursif sous la forme de représentations de dires que le locuteur rapporte, qu'ils réfèrent à ses propres paroles ou fassent écho à la parole d'autres personnages (« je me retourne et dis : - o. k., fais pas le con », p. 58 ; « elle dit, penchée sur la rivière : je ne la quitte jamais, je vais d'une berge à l'autre », p. 35). Les personnages impliqués par ces voix sont anonymes, qu'ils soient individués (l'allocutaire [p. 7]; la «salope » [p. 20]; « mama » [p. 34]; la «pute à l'air dingue » [p. 42]; les «loubards » du métro [p. 57]) ou collectifs (les "cons de Français » [p.11]; le "clan des entubeurs" [p. 19]). Dans tous les cas, ils sont faiblement indiciés, comme on l'a vu. Pour les faire parler, B.-M. Koltès utilise différentes formes de discours rapporté. En premier lieu, on trouve massivement le discours direct. Il crée une hétérogénéité énonciative par une alternance des plans d'énonciation (personnes, temps et espace) entre le discours cité et le discours citant. Selon que le locuteur raconte, commente ou conjecture, on trouve les configurations suivantes : présent de narration + présent (p. 58); imparfait + présent (p. 11) ; passé-composé + présent (p. 31); présent + présent (p. 39); imparfait + futur (p. 23) ; futur + futur (p. 27); conditionnel + futur (p. 47). Mais chez B.-M. Koltès, il y a peu d'hétérogénéité langagière dans la mesure où le registre de langue du locuteur n'est pas différent des personnages dont il rapporte le propos. Il s'ensuit qu'il est parfois difficile de distinguer les niveaux énonciatifs (dialogue ou commentaire) comme l'atteste l'exemple suivant : « pour moi, je les croyais invisibles, cachés là-haut [...] le petit clan des salauds techniques qui décident : l'usine et silence !, (et l'usine, moi, jamais !), l'usine et vos gueules! (et si la gueule, je l'ouvre ?), l'usine, vos gueules, et on a le dernier mot - et ils ont le dernier mot, le petit nombre des baiseurs qui décident pour nous» (p. 19). Le discours direct est le plus souvent introduit par un verbe de parole («dire», « demander », «crier ») avec une prédilection pour le verbe "dire » placé devant et plus rarement après le propos cité ( $«$ je me retourne et dis : - ok, fais pas le con, je vous invite» [p. 58]; «la nouvelle force c'est nous qu'elle me dit» [p. 23]). Tout aussi nombreux sont les rapports de pensées et les représentations de la parole intérieure introduits par les verbes "penser ", "se dire ", "se demander » et la tournure "avoir l'idée de », qu'ils concernent le locuteur («je pensais: le mien est ailleurs et je dois le cacher » [p. 30]; « je me suis dit : ailleurs tout est pareil » [p. 51] ; «j'ai une idée à te dire : [...] pas d'argent, pas de travail, cela n'arrange pas les choses» [p.13]) ou d'autres personnages («les putes deviennent risquées pense tout le monde» [p. 43]; «où aller, maintenant ? où aller? se dit tout le monde [...] où aller, maintenant? où aller qu'ils se demandent » [ibid.]). Il est aussi possible de signaler le discours direct par des indicateurs présents dans le cotexte (mention d'une présence humaine, sous-entendu ou présupposé d'acte de parole, mention d'un comportement verbal ou non-verbal... (« et je les entendais [...] - qu'est-ce qu'il peut bien faire» [p. 11]; «le petit clan des salauds techniques qui décident : l'usine et silence!» [p. 19]; « et quand je l'ai abordée: tu n'as pas du feu » [p. 21] ; « qui lui a donné la recette : tu vas dans le cimetière » [p. 46]...) Pour introduire la parole autre au discours direct B.-M. Koltès n'utilise pas le marquage typographique par les guillemets. Il a le plus souvent (mais pas toujours) recours à un signe de ponctuation, que ce soit les deux points ou les tirets : «je me disais : rien de plus facile que de sentir le sens du vent » (p. 29) ; « ils ne disent rien comme s'ils ne m'avaient pas vu - o. k., ne faîtes pas le con, vous me rendez mon fric [...] et on continue ensemble -, ils se regardent toujours» (p.59), voir cumule les deux : «je me retourne et dis : - ok fais pas le con, je 
vous invite [...] on ne s'emmerdera pas » (p. 58). Il n'est pas rare que le dialogue soit rapporté sans verbe introducteur, au sein de l'énonciation première ( « je suis plutôt pour faire mon coup et me barrer : ça va pour toi ? correct ! » [p. 39]) ou insérés dans des tirets (" elle ne me reconnaissait toujours pas, dans ce drôle de café - viens avec nous minet - et j'y aurais été » [p. 24]). Il est possible enfin de trouver quelques reprises en écho, qu'elles soient consensuelles (« et : correct ? correct !...», p. 41) ou dissensuelles (« l'usine et vos gueules! (et si ma gueule, je l'ouvre ?) », p. 19).

Il arrive que le locuteur ait recours au dialogue direct sous la forme de véritables scènes dialogales, comme on en trouve dans les récits conversationnels :

ils commencent à parler de plus en plus fort, pour que tout le monde entende, toujours, sans me regarder : qu'est-ce qu'il veut celui-là ? il nous cherche ou quoi ? qu'est-ce que c'est que ce mec ? pourquoi il nous les casse ? - ils me poussent vers la porte : on descend ce pédé à la prochaine station et on lui casse la gueule -, alors moi je leur dis : ok, vous me rendez mon fric, alors, et c'est bien comme cela, mais eux disent: ce pédé, qu'il attende, et on lui casse la gueule - personne ne réagit. (p. 58)

Dans tous les cas, ces dires ou pensées rapportés relèvent essentiellement du passé diégétique du personnage. À ce rappel de discours antérieurs, il faut ajouter des paroles autres du locuteur, émises sous la forme de discours factuels et contrefactuels. Relèvent de la factualité les discours qui correspondent à des propos mimétiques, en réponse à des paroles simulées, attribuées à l'allocutaire (« tu penses peut-être : pas moi, pourtant, moi je te dis : peut-être que c'est moi », p. 15) ou à un locuteur diégétique ("l'usine et vos gueules! (et si ma gueule, je l'ouvre?)»). Factuelles, encore, les paroles projetées proleptiquement dont le contenu est modalisé comme étant possible, voire certain. Le locuteur imagine ainsi les paroles qu'il prononcera lorsqu'il sera à la tête de son syndicat : «ce sera notre heure pour ne plus nous retenir camarades: faites-leur la peau maintenant, bandez, jouissez, tout ce que vous pouvez» (p. 27). Relèvent de la contrefactualité les discours présentés comme hypothétiques ( $s i$ tu le touches, il te demande pendant un kilomètre de temps si tu le touches vraiment », p. 42), potentiels (" si l'on te demande : qui est l'étranger qui est avec toi ?, tu réponds : je ne sais pas, je ne sais pas, et si on insiste, tu dis : je ne le connais pas », p. 28) ou mensongers (« je fais celui qui ne comprend pas [...] je préfère faire bien mon coup : ça colle pour toi ? o. k.!-, et me barrer, à l'aise, juste avant les grandes phrases, d'ailleurs ce coup suffit pour savoir ce qu'il y a à savoir ", p. 39). Ces simulacres de dialogue servent, comme on l'a vu à étayer une argumentation ou à concrétiser un raisonnement explicatif en liaison avec l'enjeu communicationnel du monologue.

On relèvera quelques cas de discours indirect et de discours narrativisé : « si tu le touches, il te demande pendant un kilomètre si tu le touches vraiment, avant de taper sur la gueule » (p. 42); « ils me parlaient d'un général avec ses soldats qui encerclent la forêt, làbas et qui font des cartons» (p. 50). Ils ont pour effet de linéariser et d'homogénéiser la parole du locuteur en rapprochant les discours, cité et citant, contrairement aux entailles vives de discours rapporté.

24 Au final, on dira que cette dialogie dynamise le monologue en le soumettant à une tension entre la force centripète de l'interaction immédiate engendrée par le désir de trouver refuge dans une chambre et de pouvoir s'expliquer et la force centrifuge de l'interdiscursivité qui ouvre la relation sur d'autres paroles énoncées dans d'autres contextes. 

celui qui parle avec les mots des autres. Ces rappels mémoriels, plus ou moins décelables, ont la forme d'une allusion à un dire, que la source soit identifiée ou non, ou à des données (faits, idées, valeurs) associées à des mots. C'est ainsi que l'historien détectera, par exemple, - la pièce est écrite dans le contexte de mai 1968 - un écho aux positions radicales des libertaires situationnistes (« ce sera notre heure pour ne plus nous retenir, camarades, bandez, jouissez, tout ce que vous pouvez...», p. 27). Il relèvera, autre exemple, la présence adverse, sur l'échiquier politique, des mouvements d'extrême droite, qu'ils se baptisent « Occident » ou « Ordre Nouveau » dont la mémoire, collective, comme on l'a vu dans le chapitre précédent, a gardé le souvenir de leurs " ratonnades ».

Il existe une autre forme de dialogie constitutive et latente, qui renvoie au " plurilinguisme et à la plurivocalité qui en est issue » (Bakhtine, 1978) inhérent à des pans entiers du lexique, au sens où les mots que l'on utilise emportent généralement avec eux les significations et les connotations contextuelles de leur emploi. C'est ainsi que l'on peut constater, en observant le vocabulaire prêté au personnage-locuteur, la représentation, stylistiquement construite, d'un effet d'éthos populaire sous la forme d'un locuteur de sexe masculin qui transgresse les interdits de la langue légitime comme l'attestent les grossièretés («ta gueule à toi ou je te la casse », p. 24) et les jurons (« mais merde comme un con j'ai attendu une nuit », p. 36 ; «mais merde, elle n'est pas venue, et elle ne viendra plus », p. 37). À quoi s'ajoute toute la gamme d'un style «familier » ou «non-conventionnel » (Lodge, 1992) du type "chialer», «j'en ai ma claque », « où je m'étais fourré", "une belle nana", "me balader », etc., ainsi que l'utilisation de dénominations dévalorisantes («les cons d'en bas », p. 10 ; «les pires des salauds», p. 18; "saloperie de carrefour ", p. 12 ; «le clan des salauds techniques ", p. 19). Ces dernières ont pour fonctionnalité commune la dégradation des objets du discours auxquels ils réfèrent et, à travers eux, directement ou par implication, des personnages désignés. Les grossièretés exploitent les formes carnavalesques (Bakhtine, 1970) de l'expression du bas corporel par l'intermédiaire d'obscénités principalement sexuelles ("se faire niquer", p. 25 ; «qu'on se fasse rentrer dedans », p. 20 ; «le petit clan de salauds aux gueules de tringleurs ", p. 22 ; «c'est là qu'ils nous guettent et nous baiseraient », p. 26). Populaire, encore, l'exhibition de «dispositions viriles» (Bourdieu, 1983), qu'elles se manifestent par un penchant à la violence verbale - on vient de le voir - et à la violence physique (" chez moi, on cogne tout de suite, sans gueuler, on n'est pas le genre timide », p. 42; «j'ai envie de cogner, la bonne femme là-haut accrochée à la rambarde [...] et l'Arabe qui se chante son truc pour lui tout seul, j'ai envie de le cogner ", p. 61) ou par un sexisme caractérisé. C'est ainsi que la femme, à l'exception de l'inconnue du pont, est lexicalisée péjorativement (les "salopes», p. 26; les "putes", p. 41) ou valorisée pour ses seuls attributs physiques («j'étais d'accord avec tout [...] à cause de ce qu'elle était belle comme ce n'est pas possible», p. 25 ; « je suis le mec, moi, plutôt que de parler, à suivre une belle nana », p. 55).

Ce «signum de classe » (Guiraud, 1965) entre en convergence avec la thématisation fictionnalisée de situations (émigration et marginalité citadine), d'activités (errances nocturnes [p. 41], allusion aux stupéfiants [p. 38]) et de comportements (racisme [p.11], homophobie [p.59]) auxquels se heurte le personnage-locuteur.

On retrouve les mêmes phénomènes de dialogisation dans les deux autres pièces. Je me contenterai donc d'en rendre compte brièvement. 

cesse de s'adresser à sa mère disparue («Est-ce vous qui me hantez, encombrant les carrefours, les interstices de l'existence, ou est-ce moi qui vous tiens prisonnière dans les filets du souvenir, vous interdisant de migrer vers un hypothétique repos?», p. 29). En nombre d'occurrence de propos rapportés, le dialogisme est essentiellement interdiscursif. Le narrateur rapporte à plusieurs reprises des paroles ou des écrits autres à l'aide du style direct (« Cédoine est venu ouvrir la barrière en criant - ils sont là, ils sont là ! », p. 25 ; « Je lisais les lettres, les cartes postales [...]. Les pages blanches se succédaient et tout à coup cette phrase - hier je suis rentré de Fort-Dimanche où j'ai été emprisonné pendant cinq jours », p. 23). On trouve aussi du style indirect (« Un camarade est entré. Il m'a dit qu'on me demandait au téléphone », p. 13), voire du discours narrativisé (« Nous devisions comme si nous nous étions quittés la veille », p. 40). Quant à l'autodialogisme, il est permanent et se manifeste par des commentaires et des réflexions que le narrateur s'adresse à lui-même : « Est-ce que j'invente tout cela ? Est-ce que je dis la vérité ? (p. 32).

Avec P. Kermann, on est en présence d'une polyphonie de l'au-delà qui correspond massivement aux voix des naufragés du Titanic mais résonne aussi dans les récits diégétiques, créant un effet de présence qui nous fait oublier que ce sont des disparus qui parlent. Pour ce faire, P. Kermann utilise massivement le discours rapporté direct à l'aide d'un verbe de communication introducteur (dire, ajouter, répondre) mais sans guillemets. Il arrive aussi à P. Kermann d'avoir recours à un tiret ouvrant ou à une suite de tirets pour marquer une succession de tours de paroles :

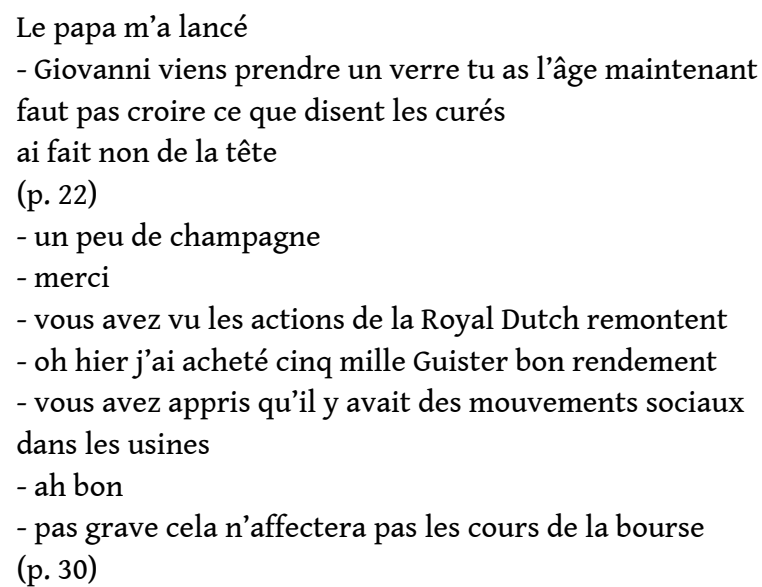

La voix fantôme du récitant rapporte du fond de la mer des voix anonymes mêlant personnels et voyageurs dans une sorte de murmure. Cette orchestration de voix multiples s'apparente à ce «silence bruissant » dont parle E. Levinas (1982) et que l'on entend quand on approche un coquillage de l'oreille, le tout symbolisant la «négativité des voix ».

la salle de gymnastique vient de fermer

et il effleure la surface plane de la mer

caresse douce

toujours plus vite

vingt et un nœuds maintenant

bientôt le quart

à nous le repos des travailleurs

chauffe

encore du charbon

pchui 


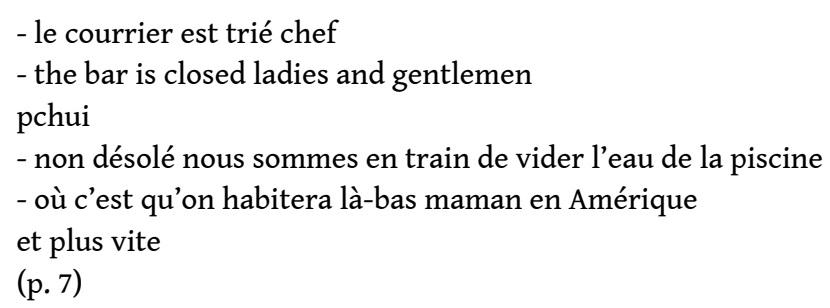

On peut noter aussi la présence de passages autodialogiques en forme de boucles réflexives qui reviennent sur son dire ou de monologues extériosés qui permettent d'accéder au flux de pensée et de conscience du personnage :

quand elle s'est retournée ai dit

oui moi Giovanni ai dit à la Mamma

maintenant il faut que je parte

(p. 22)

Alors quand ils sont entrés je me suis dit aïe qu'est-ce

qu'ils me veulent

peuvent pas me laisser finir tranquillement

surtout qu'on va bientôt sombrer

définitivement

(p. 36)

\section{Du poétique}

Ces trois pièces ont en commun le fait d'être structurées par des mouvements rythmiques de reprises thématiques et formelles. Ils correspondent, au niveau du personnage, à une pensée qui souffre à revenir sur des évènements qui l'ont traumatisé ou à un ressassement obsessionnel. Au niveau des auteurs, les répétitions relèvent d'une volonté des dramaturges d'imprimer à leur texte un rythme et une orientation stylistique proche de la prose poétique.

Chez B.-M. Koltès, par exemple, les répétitions ont une fonction compositionnelle et musicale que procure le retour de syntagmes proches ou identiques en liaison avec les isotopies dominantes du texte. Lorsqu'il n'y a pas de distance entre une occurrence et le segment répété, la répétition d'un mot a souvent une visée expressive («saloperie de quartier, saloperie d'habitude", p. 12 ; «où aller? où aller?", p. 45) ou impressive (" c'est justement là : camarade, camarade!", p. 22). Quand des structures identiques ou similaires sont reprises à moyenne ou longue distance, par contre, la répétition s'apparente à une sorte de motif qui se dissémine tout au long du texte. Il en va ainsi des allusions négatives du locuteur à ces « cons de Français " et à leur incapacité de tolérer toute forme d'altérité (« en bas sont les cons », p. 7 ; « avec les cons d'en bas », p. 10 ; « ces cons de Français se demandent », p. 11 ; « ces cons de Français sans imagination », p. 11 ; « tous les cons stationnés », p. 11 ; « tous ces cons de Français », p. 32 ; « tous ces cons de Français », p. 54 ; « comme dehors tous ces cons », p. 55).

Parmi la variété de répétitions, B.-M. Koltès a une prédilection pour les anaphores rhétoriques. Sur le plan formel, elles concernent un mot, peuvent s'étendre à une phrase ou reprendre une structure syntaxique : « tandis que ceux d'ici n'ont rien derrière la tête, prêts pour être contents, prêts pour s'amuser, prêts pour jouir tout ce qu'ils peuvent " (p. 53) ; «j'en ai ma claque, cette fois ça y est, je ne me retiens plus, j'en ai ma claque, moi, de tout ce monde-là, de chacun avec sa petite histoire dans son petit coin, de leurs gueules à tous, j'en ai ma claque de tous et j'ai envie de cogner [...] j'en ai ma claque de 
leurs gueules et de tout ce fouillis»(p. 61). Les reprises anaphoriques ont des fonctions diverses et servent, en premier lieu, à souligner l'éthos volontariste du locuteur («je suis le mec, moi, plutôt que de parler [...] je suis le mec, moi, plutôt que de regarder une nana [...] mais toi ce n'est pas pareil » [p. 56]). En second lieu, les anaphores rhétoriques ont une fonction d'argumentation. C'est ainsi que la répétition anaphorique de "zone", outre sa vertu de spécification, manifeste en jouant sur le procédé de l'accumulation, la dénonciation par le locuteur des plans des exploiteurs: «toute la série des zones que les salauds ont tracé pour nous, sur leurs plans [...] les zones de travail pour la semaine, les zones pour la moto et celles pour la drague, les zones de femmes, les zones d'hommes, les zones de pédés, les zones de tristesse, les zones de bavardage, les zones de chagrin et celles du vendredi soir » (p. 44).

Chez J.-R. Lemoine, nombreuses sont les répétitions dont les effets lyriques et pathétiques sont indéniables. Il peut s'agir d'exprimer une émotion (« Nous avons ouvert toutes les persiennes et la lumière a envahi le salon... Nous avons ouvert toutes les persiennes et la lumière a envahi le salon. C'était la douce lumière du salon », p. 21 ; «j'ai retrouvé ma sœur et ceux qui m'attendaient là les larmes, les larmes, les larmes... » p. 14). Le plus souvent, en forme de leitmotiv, la répétition traduit le sentiment d'abandon que connait le narrateur après la disparition de sa mère ( Vous me manquez, maman, vous me manquez. Je voudrais que vous soyez là », p. 17 ; «Vous me manquez », p. 24 ; «Vous me manquez, vous me manquez, je voudrais... », p. 52 ; «Je n'ai plus d'horizon. Je n'ai plus d'horizon ", p. 29). Inversement, d'autres répétitions, soulignées à l'aide de lettres capitales, manifestent la volonté du narrateur d'aller jusqu'au bout du récit de son malheur («Prendre son souffle », p. 34 ; PRENDRE SON SOUFFLE », p. 36 ; «PRENDRE SON SOUFFLE », p. 38). Quant à P. Kermann, la répétition se limite à des occurrences d'une même formule avec dislocation par une reprise pronominale du thème dans le rhème. $\mathrm{Ce}$ qui permet de mettre en valeur le personnage narrateur et de rappeler à la vie le disparu ( "moi Giovani Pastore adore les chiffres », p. 11 ; «Giovani Pastore me souviens », p. 14; "Giovani Pastore, un jour... » p. 21 ; «Giovani Pastore ai choisi les petites cuillères ", p. 26 ; « Giovani Pastore ai coulé », p. 32 ; etc.).

\section{Conclusion}

D'un point de vue générique, les trois pièces du corpus entrent dans le paradigme de ces pièces monologuées contemporaines qui proposent un personnage ordinaire, souvent emprunté à un fait divers et embrassant d'un regard le parcours de sa vie à partir de ce promontoire qu'est la séparation, voire la mort. Elles ont en commun d'appartenir à ce que J.-P. Sarrazac (2012) appelle le «théâtre de la vie» qu'il oppose au traditionnel «théâtre dans la vie ». Il ne s'agit plus de mettre l'accent sur un moment privilégié de la vie d'un personnage mais de rendre compte de son existence même. Pour compresser une vie entière dans le temps d'une représentation, ces pièces renoncent aux formes dramatiques du "théâtre dans la vie » (action au présent, successivité temporelle et causale, dialogues) et modifient en profondeur les modes de composition dramatique avec une domination de l'intrasubjectif sur les relations interpersonnelles. Ce qui se traduit par des mouvements de rétrospection (le personnage relate des épisodes de sa vie), voire d'anticipation (il se projette dans l'avenir). À quoi s'ajoutent des effets lyriques qui font que H.-T. Lehmann (2002) qualifie ces pièces de « remémoration poético-épique ». 


\section{BIBLIOGRAPHIE}

\section{Textes théâtraux}

BECKETT, S. (1978) [1975]. Cette fois. Trad. par l'auteur. Paris : Éditions de Minuit.

BECKETT, S. (1982) [1982]. Solo. Paris : Éditions de Minuit.

BILletdoux, F. (1996). Monologues. Arles : Actes Sud.

DARLEY, E. (2009). Le Mardi à Monoprix. Arles : Acte Sud.

DURIF, E. (1990). Le Petit Bois. Arles : Acte Sud.

KERMANN, P. (1999). The Great Disaster. Carnières-Morlanwelz : Lansman.

KOLTÈs, B.-M. (1988). La Nuit juste avant les forêts. Paris : Éditions de Minuit.

LEMOINE, J.-R. (2006). Face à la mère. Besançon : Éditions Les Solitaires intempestifs.

MINYANA, Ph. (1993). Chambres. Paris : éditions théâtrales.

\section{Textes théoriques}

ARISTOTE. (1980). La Poétique. Texte, traduction, notes par R. Dupont-Roc et J. Lallot. Paris :

Éditions du Seuil.

AUTHIER-REVUZ, J. (2004). « La représentation du discours autre : un champ multiplement hétérogène ». In : Muñoz, J. M., Marnette, S. \& Rosier, L. (éds), Le Discours rapporté dans tous ses états. Paris : L'Harmattan, p. 35-53.

BAKHTine, M. (1970). L'ÆEuvre de François Rabelais et la culture populaire au Moyen-Âge et sous la Renaissance. Paris : Gallimard.

BAKHTINE, M. (1978) [1975]. « Du discours romanesque ». In : Esthétique et théorie du roman. Trad. du russe par D. Olivier. Paris : Gallimard, p. 83-233.

BENHAMOU, A.-F. (2001). « Le lieu de la scène : quelques hypothèses sur l'œuvre de Koltès dans son rapport au plateau de théâtre ». In : Petitjean, A. (éd), Koltès, la question du lieu. Metz : CRESEF, p. 45-62.

BOURDIEU, P. (1983). « Vous avez dit "populaire” ? ». Actes de la recherche en sciences sociales 46, p. 98-105. En ligne : https://www.persee.fr/doc/arss_0335-5322_1983_num_46_1_2179.

BRES, J. (2005). « Savoir de quoi on parle : dialogue, dialogal, dialogique, dialogisme,

polyphonie... ». In : Bres, J. et al. (éds), Dialogisme et polyphonie. Approches linguistiques. Bruxelles :

De Boeck, p. 47-61.

GUIRAUD, P. (1965). Le Français populaire. Paris : Presses universitaires de France.

LEHMANN, H.-T. (2002) [1999]. Le Théâtre postdramatique. Trad. de l'allemand par P.-H. Ledru. Paris :

Éditions de L'Arche. 
LÉVINAS, E. (1982). Éthique et infini. Paris : Fayard.

LODGE, R. A. (1992). « Le vocabulaire non standard suivant les perceptions des locuteurs français actuels ». In : Groupe d'étude en histoire de la langue française, Grammaire des fautes et français non conventionnel. Paris : Presses de l'École normale supérieure, p. 341-354.

PIEMME, J.-M. (1994). « Entretien ». Alternatives théâtrales 45, p. 48.

SARRAZAC, J.-P. (1989). Théâtres intimes. Arles : Actes Sud.

SARRAZAC, J.-P. (1996). « Koltès, la traversée du théâtre ». Théâtre aujourd'hui 5, p. 58-65.

SARRAZAC, J.-P. (2012). Poétique du drame contemporain. Paris : Éditions du Seuil.

SCHERER, J. (1950). La Dramaturgie classique en France. Paris : Nizet.

SZONDI, P. (1983) [1956]. Théorie du drame moderne. Trad. de l'allemand par P. Pavis avec la collab. de J. et M. Bollack. Lausanne : Éditions L'Âge d'homme.

TRIAU, C. (2009). «Le personnage, entre imposition et subversion : usage du monologue dans le théâtre français du XVII ${ }^{\mathrm{e}}$ siècle ». In : Dubor, F. \& Triau, C. (éds), Monologuer. Pratiques du discours solitaire au théâtre. Rennes : Presses universitaires de Rennes, p. 111-142.

UBERSFELD, A. (1999). Bernard-Marie Koltès. Arles : Actes Sud.

\section{RÉSUMÉS}

Depuis quelques décennies, on assiste à une véritable vogue de la parole solitaire au théâtre (monologues ou soliloques) sous la forme d'un personnage, seul en scène, qui parle et raconte. Ces longues coulées verbales ont pour effet de remettre en cause les grands principes des pièces dramatiques (unicité de l'intrigue, progression ordonnée et finalisée des actions, développement temporel chronologique, causalité explicite des enchainements). C'est ainsi que dans un premier temps, je montrerai en quoi ces textes sont de véritables récits, car la fable, proche en cela des fictions romanesques, n'est plus soumise au développement temporel strictement chronologique. Dans un second temps, on verra que la solitude du personnage n'est pas forcément antidramatique puisque ces monologues sont très polyphoniques en faisant advenir de nombreux personnages et c'est là un indice du processus de romanisation que connait le théâtre depuis plusieurs années.

For some decades, we have seen a real vogue of solitary speeches in theatre (monologues or soliloquies) by the displays of a character, speaking and telling stories, alone on stage. These long verbal flows are aimed at questioning the main principles of dramatic plays (uniqueness of the plot, orderly and finalized progression of the actions, the chronological development and the explicit causality of the deployment of actions). I therefore, in the first instance, will show how these texts are indeed true tales because the fable, close in this to fictional fiction, is no longer limited to the temporal progression strictly chronological. In a second step, I will show that the solitude of the character is not inevitably anti-dramatic because these monologues are very polyphonic in making many characters happen in view of the multiple characters. It a sign of process of romanization that the theater has been experiencing for several years. 
INDEX

Mots-clés : monologues, narration dramatique, dialogisme

Keywords : monologues, dramatic narrations, dialogism

\section{AUTEUR}

ANDRÉ PETITJEAN

Université de Lorraine, Crem, F-57000 Metz, France 